\title{
-Note- \\ Treponemes-Infected Canker in a Japanese Racehorse: Efficacy of Maggot Debridement Therapy
}

\author{
Atsutoshi KUWANO ${ }^{1 *}$, Hidekazu NIWA ${ }^{2}$, Tohru HIGUCHI ${ }^{3}$, \\ Hideya MITSUI ${ }^{4}$ and Robert A. AGNE ${ }^{5}$
}

${ }^{1}$ Equine Research Institute, Japan Racing Association, 321-4 Tokami-cho, Utsunomiya-shi, Tochigi 320-

0856, ${ }^{2}$ Equine Research Institute, Epizootic Research Center, Japan Racing Association, 1400-4 Shiba, Shimotsuke-shi, Tochigi 329-0412, ${ }^{3}$ Hidaka Agriculture Mutual Aid Association, 200 Higashihourai, Mitsuishi, Shinhidaka-cho, Hidaka-gun, Hokkaido 059-3105, ${ }^{4}$ Division of Cardiovascular Surgery, Tsukazaki Hospital, 68-1, Waku, Aboshi-ku, Himeji, Hyogo 671-1227, Japan, ${ }^{5}$ Rood and Riddle Equine Hospital, 150 Georgetown Road \& Lexington, Kentucky 40511, U.S.A

\begin{abstract}
A 3-year-old thoroughbred colt presented with canker on its left hind foot. Subsequent development of cottage cheese-like horns and dermatitis disturbed healing, despite the use of miscellaneous orthodox treatment approaches to the lesions. Histological examination revealed exudative and suppurative dermatitis, and proliferatively suppurative epidermitis infected with helically coiled treponemes. Total debridement under general anesthesia led to a temporary improvement, but the ground surface regenerated abnormal epidermis similar to that observed initially after surgery. Maggot debridement therapy (MDT) was attempted, which removed all the abnormal tissue. After MDT, general farriery trimming helped to correct the distorted ground surface, and the horse returned to constant training and eventually raced. This case shows that MDT was successfully used for treatment of an intractable and treponemes-infected canker.
\end{abstract}

Key words: canker, horse, maggot debridement, treponemes

Canker is usually observed in horses kept in unhygienic conditions $[9,13]$; however, it can also be observed in well-maintained racehorses kept in hygienic conditions [12]. It is most often observed in the hind hoof, although any limb can be affected. This syndrome is diagnosed by its macroscopic features and a characteristic fetid odor and should be distinguished clinically from thrush and laminitis. In the early stage, it appeared to be atypical moist pododermatitis with loosened and ulcerative epidermis over the frog and its surrounding horny tissues $[1,12,13]$. Similarly, chronic lesions form characteristic papilla-like horny structures, which make canker difficult to treat, especially in neglected cases [11]. Although the etiology of canker has not been investigated extensively, because the condition is encountered infrequently, many etiological factors are speculated to be associated with its occurrence [9]. For example, irritant stimulation of the sensitive layer of the foot due to virus, bacteria involving spirochete-like microorganisms, fungi, or immunological

This article was accepted June 18, 2012

*Corresponding author. e-mail: hoofvet@center.equinst.go.jp
J. Equine Sci.

Vol. 23, No. 3

pp. $41-46,2012$ etiology were thought to be preferential factors, especially in unsanitary or wet environments [9-12]. Meanwhile, the pathognomonic condition of canker is known to be very similar in its clinical course and pathological appearance to bovine digital dermatitis (DD), of which abnormal feet, especially in the interdigital region, could be diseased by treponemes $[3,5,14]$. Bovine DD caused by treponemes is expected to be transmitted by contact with treponemes that contaminate the stall floors [18]. In addition, polymerase chain reaction (PCR) techniques targeting the Treponema-specific $16 \mathrm{~S}$ ribosomal RNA gene fragments (16S rDNAs) were recently used to prove that plural clone genes of noncultivatable treponemes infected lesions of the equine canker [7]. In that study, some clones in the canker coincided with the treponemes detected in bovine DD. These above reports support the opinion that treponemes could contribute to occurrence and/or worsening of canker. However, whether treponemes are infectious or opportunistic is a controversial question, to date $[11,12]$. In usual practice, mild canker is treated by topical applications over affected tissues [9]. Alternatively, in severe cases, a sharp scalpel, hoof knife, or electrocautery under 


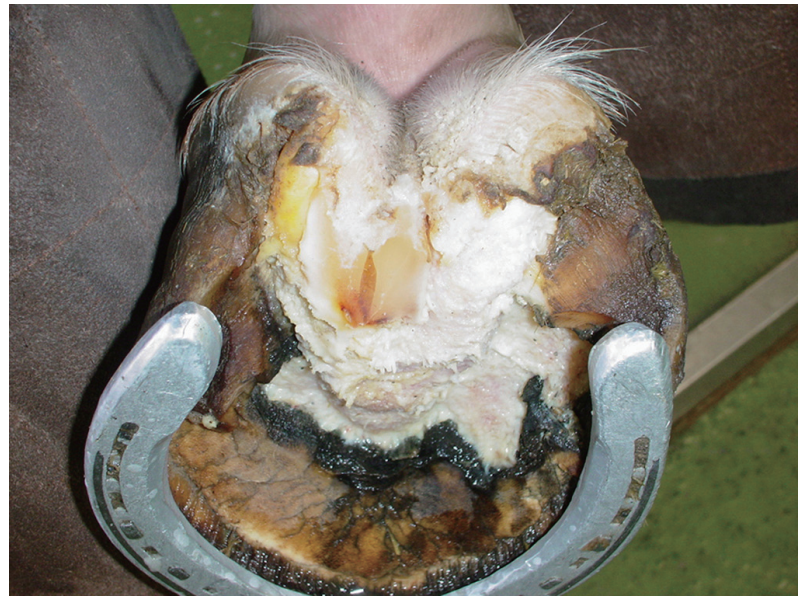

Fig. 1. The ground surface of the affected foot at a relatively early stage.

The diseased foot shows swollen dermis and custard cream-like softened horny structures extending to most of the frog and a portion of the sole on the left hind foot.

general anesthesia is used to remove proliferative and poorly cornified tissues until normal corium is revealed $[4,11]$. Although a previous report claimed good prognosis for complete resection if treatment was instituted early [20], advanced cases have usually been refractory [1]. As a relatively new therapy, medical grade sterile maggot debridement therapy (MDT) can be used for treatment of canker [8, 16, 17]. Maggots applied to canker lesions promote formation of healthy granulation tissue on which good healthy epidermis can growth [6]. It is known that maggots remove damaged tissue as well as its associated bacteria by digestive secretions, except for Proteus [8]. However, there is no evidence that maggots are effective against treponemes-infected canker, to date. A disadvantage of MDT is the difficulty in providing sufficient air to preserve the lives of the maggots when the wound is highly exudative [6]. Considering this issue, MDT is probably not suitable for severely exudative canker cases. We describe the case of a thoroughbred colt in which a treponeme-infected canker was successfully treated with MDT.

A 3-year-old thoroughbred colt initially developed a small granulomatous lesion on the medial aspect of the frog of the left hind foot and coincidently became slightly lame on the same limb. A few weeks later, the lesion developed a swollen and reddish dermis, indicating exudative dermatitis, and produced cottage cheese-like soft horny structures spreading to the frog and the sole (Fig. 1). Thus, iodine and chlorhexidine gluconate solution were used as disinfectants or an embrocation of alum solution as an astringent was initially applied to the wound of

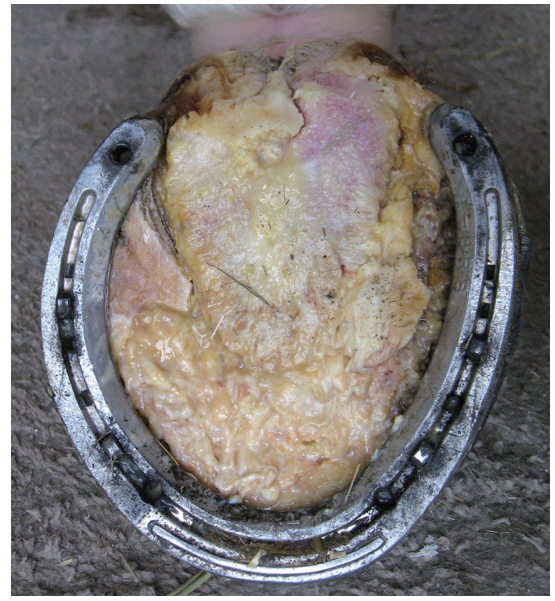

Fig. 2. Severely chronic, exudative, and proliferative pododermatitis on the entire ground surface was observed 8 months after the onset of canker.

the horse. Because progression of secondarily infectious pododermatitis was eventually observed, the referring veterinarian changed the daily systemic treatment to a combination of cephalothin sodium ( $20 \mathrm{~g} / \mathrm{head}$ IV) and gentamicin sulfate ( $1 \mathrm{~g} / \mathrm{head} \mathrm{IM})$, with topical application of a mixture of $2 \%$ cephalothin sodium and $0.1 \%$ gentamicin sulfate ointments to the hoof lesion at relatively early stages. In addition, a sharp hoof knife was used to debride the lesion, which was carefully washed with physiological saline solution, and the affected foot was dressed with sterile gauze and bandaged. Despite those miscellaneous orthodox treatments, the lesions eventually extended to the whole ground surface and showed severely chronic, exudative, and proliferative pododermatitis 8 months after the onset of the disease (Fig. 2). At this stage, the abnormal structures were radically debrided under general anesthesia by the triple-drip method [2], with the abaxial sesamoid nerve block used for local anesthesia [15]. This total resection decreased the abnormal growth and further production of exudate. However, 3 weeks after the total resection, abnormal horny structures similar to those that were observed initially had unfortunately reappeared. Hence, we decided to try an alternative treatment, MDT, to eliminate the recurrent lesions. The MDT methodology involved 600 first or second instar larvae of Phaenicia (Lucilia) sericata (Japan Maggot Company Ltd., Okayama, Japan).

The numbers of maggots applied were in accordance with human medical guidelines for MDT, according to which 7 to 8 larvae can be applied per square $\mathrm{cm}$ of wound surface area [6]. An aluminum treatment-plate shoe 


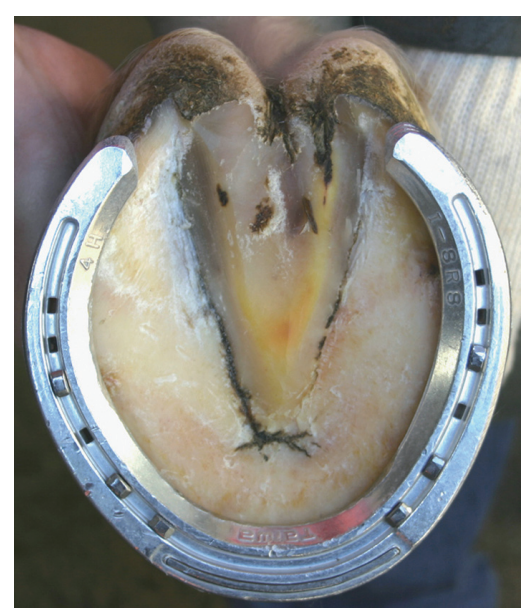

Fig. 3. Fully healed foot 4 months after the last application of maggots.

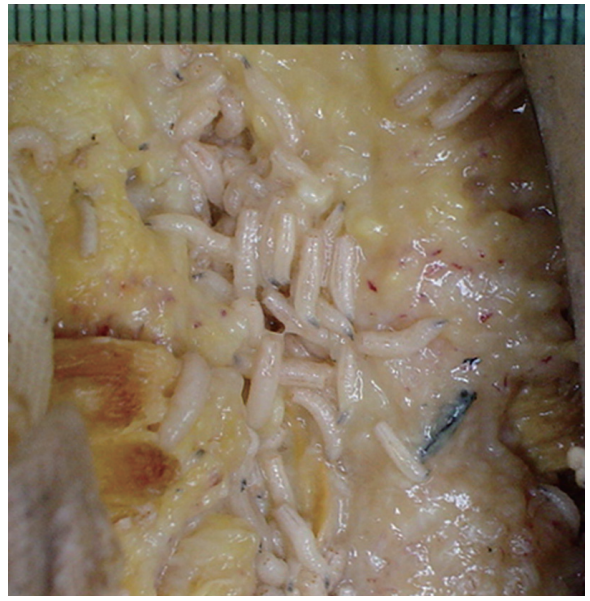

Fig. 4. The larvae grown to mature 3 days after application to the lesions. that covered the entire sole and frog was applied to the affected foot. Furthermore, the entire foot was bandaged with elastic bandaging material (Elasticon) (Sumitomo 3M Ltd., Tokyo, Japan) to help prevent contamination of the lesion. The dressing was changed daily, and after every 3 days, the mature (Fig. 4) and dead larvae that had drowned in the exudate were removed and replaced with new living larvae. During MDT, systemic and topical antibiotics were not administered to the horse. The maggots could eliminate all grossly abnormal tissues within 1 month from the beginning of MDT, after which the therapy was discontinued. Furthermore, the maggots made the subsolar surface of the hoof appear as topically black to dark-brown horny tissues indicating maggot induced horn degeneration. However, these discolored horns did not persist and gradually improved to normally appearing horny structures by means of routine farriery trimming. Four months after MDT was discontinued, the hoof had healed completely and the foot was perfectly conformed (Fig. 3). The horse returned to training and raced four times before it was retired from racing. This canker condition did not develop on the other feet or spread to other horses on the farm.

For laboratory study, diseased tissues resected from the apex and body of the frog, medial and lateral aspects of the sole, and the white line were examined histologically at 2 months, 8 months (at the total resection), 9 months (at the recurrence), 10 months (at finish of the MDT), and 14 months (completely healed) after the onset of the disease. Histopathological tissue analysis using the hematoxylin and eosin stain method of the tissues obtained at 2, 8, and 9 months after the onset of disease showed edematous change and mononuclear cell infiltration in dermis, proliferation and partial necrosis of epidermis, keratolytic degeneration like vacuolar dyskeratosis and parakeratosis, and neutrophil infiltration to the degenerated epidermis. These observations indicated that the pathological condition was exudative and suppurative dermatitis, and proliferatively suppurative epidermitis. Warthin-Starry staining of the same specimens as used in the above stages showed a large number of helically coiled spirochete-like microorganisms in the degenerated epidermis at all depths except the dermis (Fig. 5). In contrast, no spirochete-like microorganisms were observed in the healed tissues resected 14 months after the onset of disease. Gram staining showed gram-positive microorganism infestation in the microcracks and superficial border of the horny structures at all stages. No fungi were observed in any sections confirmed by periodic acidSchiff and Grocott's methenamine silver stainings. Immunohistochemical analysis using a rabbit anti-Treponema pallidum polyclonal antibody (DAKO Japan Inc., Kyoto, Japan) showed antigenicity against spirochete-like microorganisms in the epidermis (Fig. 6). This result revealed that the spirochete-like microorganisms observed in the Warthin-Starry staining were treponemes. Transmission electron microscopy (Hitachi H7600) (HITACHI High Technologies, Tokyo, Japan) of the tissues infected with spirochete-like microorganisms showed that many electron-dense spirillar bacteria with 2 to 8,100 - to $200-\mathrm{nm}$ diameter axes between their outer membrane sheaths and cell cylinders were present within the intercellular spaces and cytoplasm of degenerated keratinocytes (Fig. 7). This electron microscopic morphology of the infected microorganism coincided with the characteristics of Treponema. In adddition, the manufactured DNA isolator (PS-rapid 


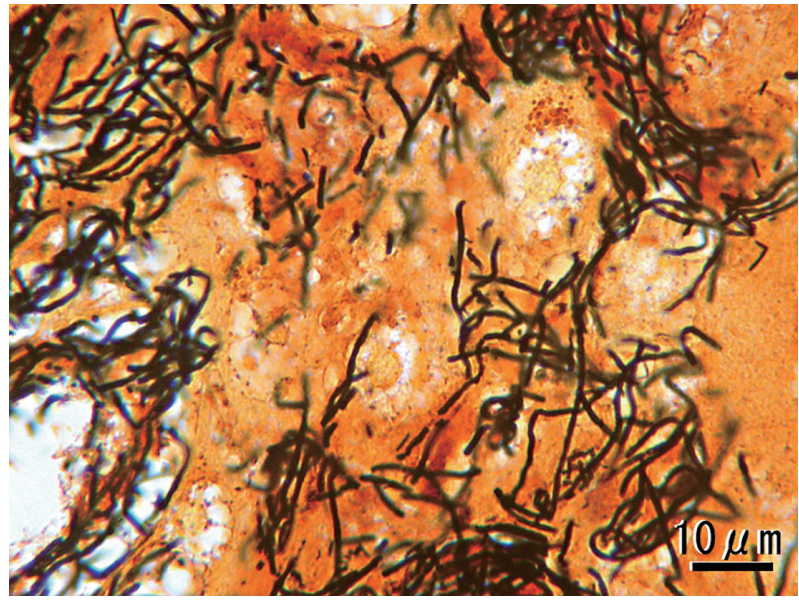

Fig. 5. In the Warthin-Starry stain, silver-stained black spirochete-like microorganisms can be seen in the dyskeratotic epidermis. Bar $=10 \mu \mathrm{m}$.

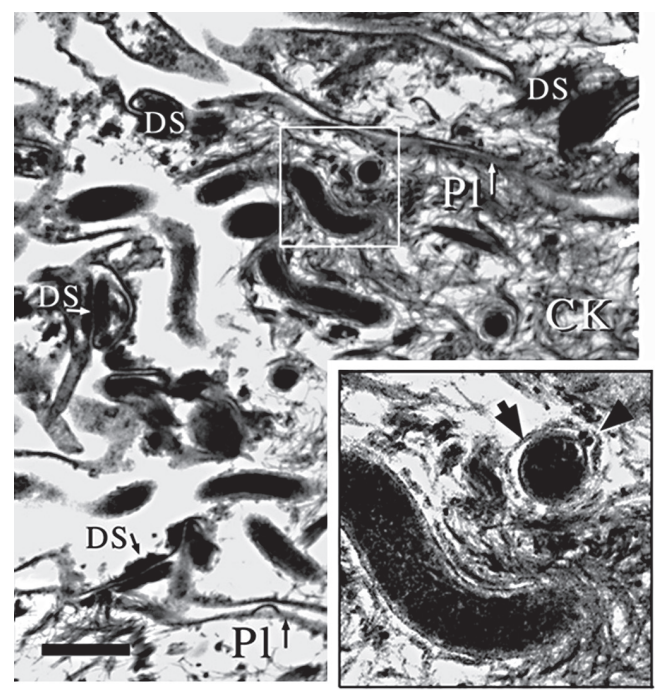

Fig. 7. Transmission electron microscopic observation.

Treponemes possessing pathognomonic axes (arrow head in the inset) between the cell wall and outer membrane sheath (arrow in the inset) are shown. The microorganisms exist in both the degenerative keratinocytes and intercellular spaces. This observation is corresponding to the totally debrided specimen 8 months after the onset. CK: cytokeratin filaments, $\mathrm{Pl}$ : plasmalemma, DS: desmosome. Bar=500 nm.

reagent) (WAKO Pure Chemical Industries, Ltd., Osaka, Japan) was used to extract the bacterial DNA from the paraffin-waxed tissue sections obtained at total resection,

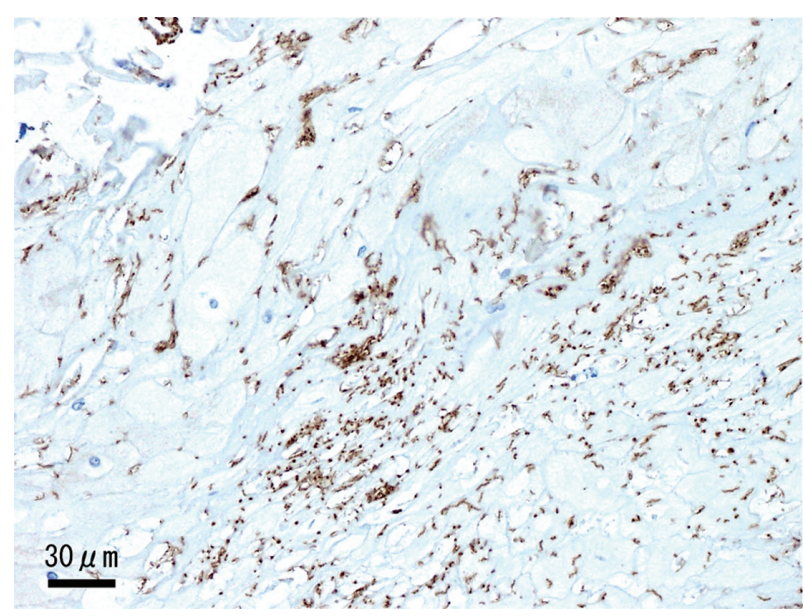

Fig. 6. Positive reaction to immunohistochemical analysis using the anti-Treponema pallidum polyclonal antibody indicates that the spirochete-like microorganisms are Treponema species.

This observation is corresponding to the recurrent lesion. Bar $=30 \mu \mathrm{m}$.

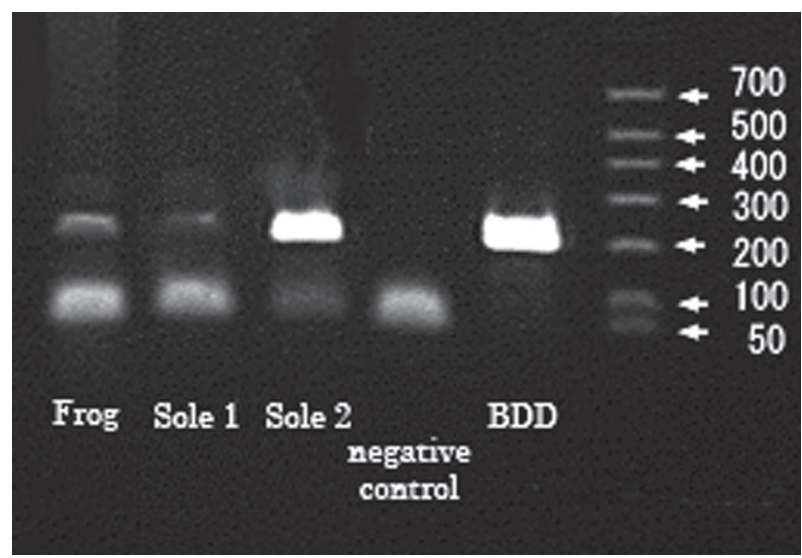

Fig. 8. Polymerase chain reaction results for the treponemespecific 16S rDNAs of the lesions (Frog, Sole 1, Sole 2), negative control (rDNAs derived from sound hoof tissue), and the positive control (Treponema $16 \mathrm{~S}$ rDNAs derived from bovine digital dermatitis).

The affected tissues and a positive control show the same size of DNA; approximately 200-base pair structures bands are observed.

recurrence, and when completely healed. Subsequently, Treponema species-specific touchdown PCR targeting bacterial $16 \mathrm{~S}$ rDNAs was performed as previously described [14] to species present at 2 months after the onset, total resection, recurrence, and healing. Consequently, specific PCR products (approximate 200-base pair structures) were identified only in the sections in 
which the spirochete-like microorganisms were observed and the positive control (Fig. 8).

The above laboratory findings suggested that the diseased condition was intimately associated with the treponemes-infection. It has been known that the peptidoglycan of treponemes could stimulate immune cells to produce several inflammatory mediators, such as cytokines and chemokines, and eventually induce inflammation [19]. Even in this canker, inflammation of the dermis occurred despite the fact that the treponemes existed only in the epidermis. It was considered that the treponemes in the epidermis produced some inflammatory mediators that passed through the epidermis to the dermis and induced the dermatitis. Injury to epidermal tissue of the foot, such as from trauma, thrush, or laminitis, may be required for invasion of canker-contributing treponemes [11]. Considering this, we thought that the recurrence was caused from reinfection by treponemes, which remained deep within the cracks or nail holes of the hoof wall after the total debridement. Total debridement also possibly caused precursor wounds that led to the recurrence. Medical treatments, including local and systemic administration of antibiotics or disinfectants, were ineffective in resolving this canker condition; the reason is not understood, but it is possible that the antibiotics and disinfectants were incapable of contacting the infecting treponemes in the epidermis or were ineffective against these treponemes. This suggested that perfect disinfection of the hoof is very difficult. Meanwhile, MDT successfully eliminated the abnormal horny structures and reduced the severe epidermitis and dermatitis. Even though this case of canker was severely exudative, which caused the maggots to drown periodically, MDT was successful because of daily dressing changes and replacement of the maggots every 3 days. The differences in the pathognomonic and laboratory findings of pre-MDT and those after successful outcome suggested that MDT could have been responsible for elimination of the cause of canker and the treponemal infection. We recommend that intractable and treponemes-infected canker cases should be resolved not only by surgical debridement but also by MDT and maintaining the horses in a hygienic environment.

\section{Acknowledgements}

We thank Dr. Norio Yamagishi from the agricultural faculty of Iwate University for providing the DNA extract obtained from bovine DD infected with treponemes. We also acknowledge Mr. Takashi Sekiguchi and Mr. Yasutaka Nakajima, who are farriers from the Japan Racing Association, for their dedicated services.

\section{References}

1. Baxter, G.M., Stashak, T.S., Belknap J.K., and Parks, A. 2002. Canker. pp. 519-521. In: Adams and Stashak's Lameness in Horses, 6th ed. (Baxter, G.M. ed.), Wiley-Blackwell Publishing, West Sussex.

2. Benson, G.J., and Thurmon, J.C. 1990. Intravenous anesthesia. Vet. Clin. North Am. Equine Pract. 6: 513-528.

3. Blowey, R.W., Done, S.H., and Cooley, W. 1994. Observations on the pathogenesis of digital dermatitis in cattle. Vet. Rec. 135: 115-117.

4. Booth, L., and White, D. 2007. Pathologic conditions of the external hoof capsule. pp. 224-252. In: Equine Podiatry, (Floyd, A.E., and Mansmann, R.A. eds.), Saunders Elsevier, St. Louis.

5. Collighan, R.J., and Woodward, M.J. 1997. Spirochaetes and other bacterium species associated with bovine digital dermatitis. F.E.M.S. Microbiol. Lett. 156: $37-41$.

6. Fleischmann, W., Grassberger, M., and Sherman, R. 2006. Maggot Therapy. A Handbook of MaggotAssisted Wound Healing (Translation of the English edition), Thieme New York, NY.

7. Moe, K.K., Yano, T., Kuwano, A., Sasaki, S., and Misawa, N. 2010. Detection of Treponemes in canker lesions of horses by $16 \mathrm{~S}$ rRNA clonal sequencing analysis. J. Vet. Med. Sci. 72: 235-239.

8. Morrison, S.E. 2005. How to use sterile maggot debridement therapy for foot infections of the horse. A.A.E.P. Proceeding 51: 461-464.

9. Moyer, W.A. 1999. Canker. pp. 1544-1546. In: Equine Medicine and Surgery, 5th ed. (Colahan, P.T., Mayhew, I.G., Merritt, A.M. and Moore, J.N. eds.), Mosby, St. louis.

10. Nagamine, C.M., Castro, F., Buchanan, B., Schumacher, J., and Craig, L.E. 2005. Proliferative pododermatitis (canker) with intralesional spirochetes in three horses. J. Vet. Diagn. Invest. 17: 269-271.

11. O'Grady S.E., and Madison, J.B. 2004. How to treat equine canker. A.A.E.P. Proceeding 50: 202-205.

12. Oosterlinck, M., Deneut, K., Dumoulin, M., Gasthuys, F., and Pille, F. 2011. Retrospective study on 30 horses with chronic proliferative pododermatitis (canker). Equine Vet. Educ. 23: 466-471.

13. Reeves, M.J., Yovich, J.V., and Turner, A.S. 1989. Miscellaneous conditions of the equine foot. Vet. Clin. North Am. Equine Pract. 5: 221-242.

14. Rijpkema, S.G., David, G.P., Hughes, S.L., and Woodward, M.J. 1997. Partial identification of spirochaetes from two dairy cows with digital dermatitis by polymerase chain reaction analysis of the $16 \mathrm{~S}$ ribosomal 
RNA gene. Vet. Rec. 140: 257-259.

15. Schumacher, J., Schumacher, J., Schramme, M.C., DeGraves, F., and Smith, R.K.W. 2007. Diagnostic analgesia of the equine foot. pp. 128-140. In: Equine Podiatry. (Floyd, A.E., and Mansmann, R.A. eds.), Saunders Elsevier, St. Louis.

16. Sherman, R.A., and Wyle, F.A. 1996. Low-cost, lowmaintenance rearing of maggots in hospitals, clinics, and schools. Am. J. Trop. Med. Hyg. 54: 38-41.

17. Sherman, R.A., Morrison, S.E., and Ng, D. 2007. Maggot debridement therapy for serious horse wounds - A surbey of practitioners. Vet. Rec. 174: 86-91.
18. Shibahara, T., Ohya, T., Ishii, R., Ogihara, Y., Maeda, T., Ishikawa, Y., and Kadota, K. 2002. Concurrent spirochaetal infections of the feet and colon of cattle in Japan. Aust. Vet. J. 80: 497-502.

19. Tanabe, S.I., Bodet, C., and Grenier, D. 2008. Treponema denticola peptidoglycan induces the production of inflammatory mediators and matrix metalloproteinase 9 in macrophage-like cells. J. Periodontal. Res. 44: 503-510.

20. Wilson, D.G. 1994. Topical metronidazole in the treatment of equine canker. A.A.E.P. Proceeding. 40: 49. 